\title{
Positioning in Chinese Digital Television Network Using TDS-OFDM Signals
}

\author{
Linglong Dai, Zhaocheng Wang and Changyong Pan \\ Tsinghua National Laboratory for Information \\ Science and Technology, Tsinghua University \\ E-mail: d1107@mails.tsinghua.edu.cn
}

\author{
Sheng Chen, Fellow, IEEE \\ School of Electronics and Computer Science \\ University of Southampton \\ E-mail: sqc@ecs.soton.ac.uk
}

\begin{abstract}
Due to wide coverage and high transmission power of digital television (DTV) transmitters, DTV-based wireless positioning is a promising complementary to global positioning system. For Chinese DTV broadcasting network whose key technology is time-domain synchronous orthogonal frequency division multiplexing (TDS-OFDM), this paper proposes a timefrequency joint positioning scheme by utilising TDS-OFDM signal properties in both the time and frequency domains. The proposed scheme needs no modification of current infrastructures, and has no impact on the normal TV program reception. Simulation results show that the positioning accuracy of less than $0.1 \mathrm{~m}$ can be achieved when the signal-to-noise ratio is higher than $20 \mathrm{~dB}$ over the realistic simulated channels.
\end{abstract}

\section{INTRODUCTION}

Compared with global positioning system (GPS) signals, digital television (DTV) signals have much higher received power, lower operating frequency, wider signal bandwidth, as well as lower Doppler effect [1]. Thus DTV-based positioning has inspired wide research interests to provide a promising positioning solution in dense urban and indoor environments where GPS performs badly or even fails to work.

Several investigations of the DTV-based positioning can be found in the literature. The positioning scheme by inserting orthogonal Kasami sequences into normal TV signals was proposed in [2] for American DTV standard set forth by the advanced television systems committee (ATSC), but it required some modifications of the current DTV infrastructure. The positioning method based on correlation of frequency-domain pilots was presented for European digital video broadcastingterrestrial (DVB-T) systems, and the achievable accuracy was several meters [3]. To the best of the authors' knowledge, there is no literature addressing the positioning issue for Chinese DTV standard whose key technology is the time-domain synchronous orthogonal frequency division multiplexing (TDSOFDM) [4]. Filling up this gap is the motivation of this paper.

The state-of-the-art positioning methods using OFDM signals can generally be divided into two categories. The first one uses the traditional or improved correlation-based timing synchronization algorithms to locate the OFDM block boundaries [5]. The positioning schemes used for DTV systems, including ATSC and DVB-T [2], [3], all belong to this category. These schemes have low complexity but their accuracies are directly limited by sampling rate, and generally an error of several meters is expected. The methods of the other category are based on super resolution algorithms, such as the multiple signal classification (MUSIC) [6], derived from modern spectral estimation techniques. These methods use super resolution algorithms to extract a more accurate estimate of the first arrived path. They have better positioning accuracy but their complexity is high, which might be unacceptable for commercial receivers.

This paper presents the complete positioning scheme using TDS-OFDM signals for Chinese DTV system in singlefrequency network (SFN). The contribution of this paper is twofold. Firstly, for transmitter identification and transmitterspecific time of arrival (TOA) estimation, it is proposed to regard transmission parameter signalling (TPS) embedded in TDS-OFDM signal as frequency-domain pilots, and then time-division multiplexing (TDM) of TPS is proposed to provide orthogonal frequency-domain pilots for TDS-OFDM signals. Secondly, the time-frequency positioning algorithm using the time-domain pseudorandom noise $(\mathrm{PN})$ sequence and the frequency-domain pilots (TPS) of TDS-OFDM signals is proposed, which achieves higher accuracy than the currently available solutions, while imposing a low complexity.

The remainder of this paper is organized as follows. Section II illustrates the positioning model in the TDS-OFDMbased SFN. Section III proposes the time-frequency joint positioning algorithm using the time-domain $\mathrm{PN}$ sequence and frequency-domain TPS embedded in TDS-OFDM signals. Simulation results are presented in Section IV, and we conclude this paper in Section V.

\section{TDS-OFDM BASEd Positioning Model}

Most DTV systems are deployed as SFN to achieve better coverage and higher spectral efficiency, whereby several transmitters simultaneously send identical signals in a synchronised way [7]. For example, $6 \mathrm{SFN}$ transmitters are deployed in the TDS-OFDM-based DTV system in Hong Kong, China [8].

The positioning model using time difference of arrival (TDOA) technique in TDS-OFDM-based SFN is shown in Fig. 1. TDOA is the difference of two TOAs corresponding to two transmitters. TDOA can remove the common TOA estimation error caused by the non-ideal synchronization between the receiver and transmitters. The prerequisite of synchronicity among different transmitters in SFN naturally provides the same time basis for every transmitter, which is 


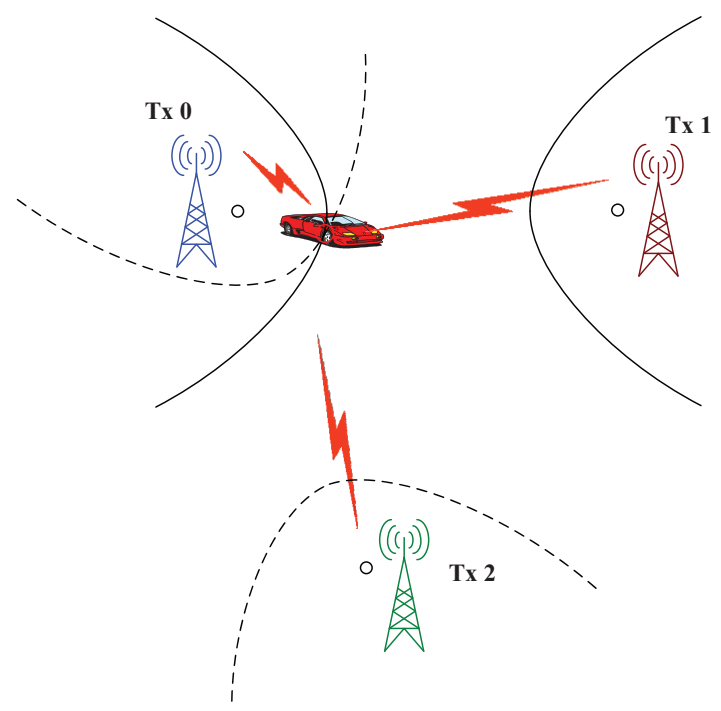

Fig. 1. Positioning using TDOA in the TDS-OFDM-based SFN.

required by TDOA technique. The receiver location can be uniquely calculated as the intersection of several hyperbolic curves corresponding to different TDOAs. It is clear that the fundamental issues for positioning in this model are accurate TOA estimation and transmitter identification.

Fig. 2 presents the TDS-OFDM signal frame structure. PN sequence is used as guard interval as well as training sequence for fast synchronization and accurate channel estimation. Therefore, frequency-domain pilots used in standard OFDM can be removed to increase the spectral efficiency by about $10 \%$ to $15 \%$ [8]. Inverse discrete Fourier transform (IDFT) block with length of 3780 consists of the normal TV program data with length of 3744 and the TPS with length of 36. TPS is used to convey system information, such as constellation mapping scheme, coding rate and interleaving mode. The 36 TPS symbols are equally divided into two groups located at two sides of the IDFT block. TPS takes BPSK modulation for reliable reception [4].

\section{Positioning Using TDS-OFDM Signals}

This section proposes the time-frequency joint TOA estimation algorithm, whose performance is also analyzed.

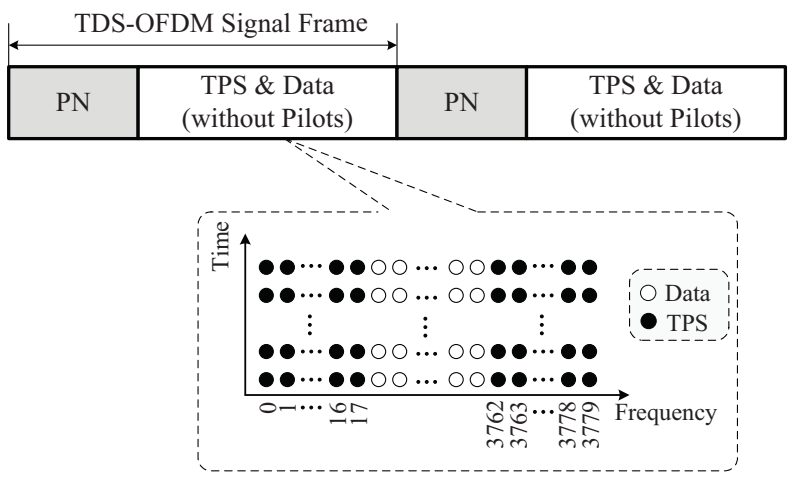

Fig. 2. TDS-OFDM signal frame structure.

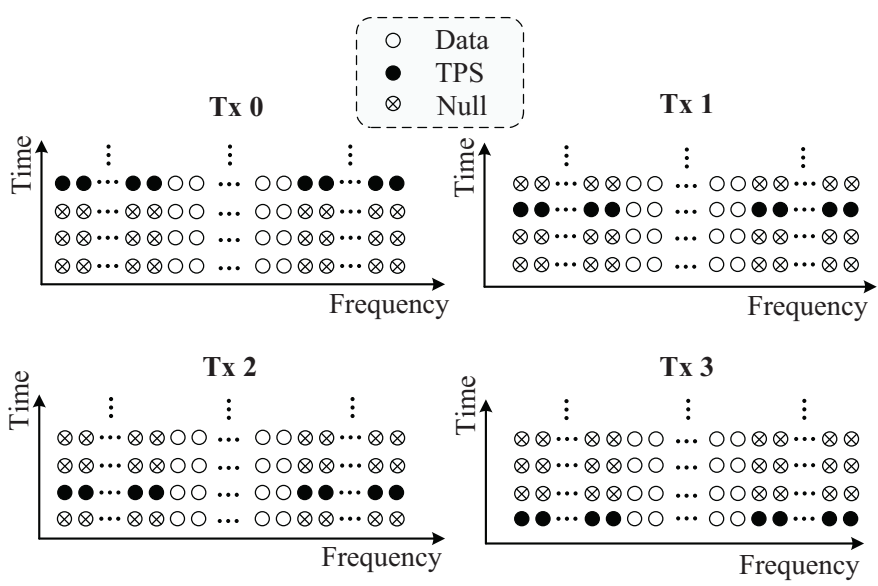

Fig. 3. TDM of TPS for positioning in the TDS-OFDM-based SFN.

\section{A. TDM of TPS as Orthogonal Frequency-Domain Pilots}

Conventionally, TDS-OFDM is regarded as a scheme without frequency-domain pilots, and PN sequence provides the only training information. TPS is only used to provide demodulation and decoding information at the receiver. However, we propose to consider TPS as frequency-domain pilots due to the invariant property of TPS. As the system information, TPS does not change frequently. Typically, TDS-OFDM broadcasting system uses the same TPS for several hours, or even dozens of days. Once the TPS with robust BPSK modulation scheme has been correctly detected in the previous signal frame, it can be regarded as known symbols in the following signal frames, whereby TPS detection is unnecessary any more. This novel look at TDS-OFDM signal frame structure provides a new degree of freedom for TDS-OFDM receiver design, as well as for positioning.

For the purpose of transmitter identification and transmitterspecific TOA estimation, TDM of TPS is then proposed in the TDS-OFDM-based SFN, which can be defined by

$$
P_{m, k}^{(i)}=\left\{\begin{array}{cc} 
\pm 1, & (i-m) \bmod (M-1)=0, k \in \Psi, \\
0, & \text { others, }
\end{array}\right.
$$

where $P_{m, k}^{(i)}$ denotes the TPS over the $k$ th subcarrier for the $m$ th transmitter in the $i$ th signal frame, and

$$
\Psi=\{0,1, \cdots, Q\} \cup\{\Delta, \Delta+1, \cdots, \Delta+Q\}
$$

is the index set of TPS, while $Q=17$ and $\Delta=3762$ according to [4].

Without the loss of generality, the time-division multiplexed TPS in the case of four transmitters are illustrated in Fig. 3. TDM of TPS provides orthogonal frequency-domain pilots for TDS-OFDM signals, offering the orthogonality for transmitter identification and TOA estimation using TPS.

\section{B. Time-Frequency TOA Estimation Algorithm}

The hierarchical frame structure specified in TDS-OFDM system ensures that each signal frame is exactly aligned with the absolute time [4]. The transmitting instant of each signal frame is known to the receiver if frame number is detected. 
Therefore, TOA can be easily obtained by finding the correct starting point of the received TDS-OFDM signal frame.

Assume that there are $M$ visible transmitters in SFN. The transmitted time-domain IDFT block of the $m$ th transmitter is

$$
x_{m}(n)=\frac{1}{\sqrt{N}} \sum_{k=0}^{N-1} X_{m, k} e^{j \frac{2 \pi}{N} k n}, 0 \leq n \leq N-1,
$$

where $N$ is the IDFT block length, $X_{m, k}$ is the frequencydomain symbol over the $k$ th subcarrier for the $m$ th transmitter.

The channel between the $m$ th transmitter and the receiver can be modelled as

$$
h_{m}(n)=\sum_{l=0}^{L_{m}-1} h_{m, l} \delta\left(n-n_{m, l}\right),
$$

where $\delta(n)$ is the Kronecker delta function, $L_{m}$ is the number of paths, $h_{m, l}$ and $n_{m, l}$ are the attenuation coefficient and delay of the $l$ th path, respectively. The transmission delay $\tau_{m, d}$ associated with the signal from the $m$ th transmitter can be normalised by the sampling period $T_{s}$ as

$$
\theta_{m}=\tau_{m, d} / T_{s}=\theta_{m, I}+\theta_{m, F},
$$

where $\theta_{m, I}$ is the normalised integral delay and $\theta_{m, F} \in$ $(-0.5,+0.5]$ is the normalised fractional delay. Note that $N T_{s}$ is the IDFT block duration in TDS-OFDM systems.

After passing through the multi-path channels, the received signal coming from the $M$ visible transmitters in the SFN is

$$
\begin{aligned}
y(n)= & \frac{1}{\sqrt{N}} \sum_{m=0}^{M-1} \sum_{l=0}^{L_{m}-1} \sum_{k=0}^{N-1} h_{m, l} X_{m, k} e^{j \frac{2 \pi}{N} k\left(n-n_{m, l}\right)} \\
& \times e^{-j \frac{2 \pi}{N} k \theta_{m, I}} e^{-j \frac{2 \pi}{N} k \theta_{m, F}}+v(n)
\end{aligned}
$$

where $v(n)$ is the additive white Gaussian noise (AWGN). After the discrete Fourier transform (DFT), the frequencydomain signal over the $k$ th subcarrier is obtained as

$$
Y_{k}=\sum_{m=0}^{M-1}\left(H_{m, k} X_{m, k} e^{-j \frac{2 \pi}{N} k \theta_{m, F}}\right) e^{-j \frac{2 \pi}{N} k \theta_{m, I}}+V_{k},
$$

where $0 \leq k \leq N-1, V_{k}$ is the DFT of $v(n)$, and $H_{m, k}$ is the channel frequency response (CFR) defined by

$$
H_{m, k}=\sum_{l=0}^{L_{m}-1} h_{m, l} e^{-j \frac{2 \pi}{N} k n_{m, l}}
$$

which is the DFT of $h_{m}(n)$ in (4).

It is clear that the impact of the normalised transmission delay $\theta_{m}$ on the received signal is twofold: time shift by $\theta_{m, I}$ samples in the time domain and linear phase rotation by $-\frac{2 \pi}{N} k \theta_{m, F}$ in the frequency domain.

Integral TOA estimation using time-domain PN sequence: The integral delays corresponding to different transmitters can be roughly estimated by the time-domain sliding correlation [9] between the received PN sequence $r(n)$ and the local PN sequence $p(n)$

$$
\operatorname{Corr}(d)=\sum_{n=0}^{N_{p}-1} r^{*}(n-d) p(n)
$$

where $N_{p}$ is the length of the PN sequence, $r^{*}(n)$ denotes the conjugate of $r(n)$, and $\operatorname{Corr}(d)$ is the correlation corresponding to the slide of $d$. Due to the near-ideal autocorrelation property of the PN sequence, the correlation peak is confirmed when the local PN sequence is aligned with the received PN sequence. Thus, correlation peaks appear at the slide values

$$
d_{m}=\theta_{m, I}, \quad 0 \leq m \leq M-1 .
$$

According to [9], the correlation-based method (8) yields the timing accuracy within the range $\left(-T_{\text {sample }} / 2,+T_{\text {sample }} / 2\right]$, where $T_{\text {sample }}$ is the actual sampling period used. In TDSOFDM receivers, the received signal is usually over-sampled by a factor of four, i.e. $T_{\text {sample }}=T_{s} / 4$, which results in the timing accuracy in the range of $\left(-T_{s} / 8,+T_{s} / 8\right]$.

Without the loss of generality, $d_{0}=\theta_{0, I}$ is assumed to be the location of the first significant correlation peak. After compensating for the first large integral delay $\theta_{0, I}$, the received DFT signal block becomes

$$
Y_{k}=\sum_{m=0}^{M-1}\left(H_{m, k} X_{m, k} e^{-j \frac{2 \pi}{N} k \theta_{m, F}}\right) e^{-j \frac{2 \pi}{N} k \bar{\theta}_{m, I}}+V_{k},
$$

where $\bar{\theta}_{m, I}=\theta_{m, I}-\theta_{0, I}$ is the differential integral delay of the $m$ th transmitter with respect to the first integral delay $\theta_{0, I}$.

However, the number of correlation peaks in (8) is usually larger than the number of visible transmitters in multi-path scenarios. Moreover, there is no way to distinguish the coincided correlation peaks corresponding to different transmitters having the same TOA. These problems can be solved by using the proposed time-division multiplexed TPS as follows.

Owing to the proposed TDM of TPS defined by (1), the received TPS $Y_{k}^{(i)}$ in the $i$ th signal frame can be rewritten as

$$
\begin{array}{r}
Y_{k}^{(i)}=\sum_{m=0}^{M-1}\left(H_{m, k}^{(i)} P_{m, k}^{(i)} e^{-j \frac{2 \pi}{N} k \theta_{m, F}}\right) e^{-j \frac{2 \pi}{N} k \bar{\theta}_{m, I}}+V_{k}= \\
\left\{\begin{array}{cc}
H_{m, k}^{(i)} P_{m, k}^{(i)} e^{-j \frac{2 \pi}{N} k\left(\bar{\theta}_{m, I}+\theta_{m, F}\right)}+V_{k}, & i=u M+m, \\
V_{k}, & \text { others, }
\end{array}\right.
\end{array}
$$

where $u$ is an arbitrary positive integer, $k \in \Psi$ and $H_{m, k}^{(i)}$ the CFR in the $i$ th frame. It is clear from (11) that TPS of the $m$ th transmitter is received at the $(u M+m)$ th signal frame.

When investigating the impact of transmission delay on the received signal, channel state information can be assumed to be known [10]. Thus the received TPS associated with the $m$ th transmitter can be simplified as below by removing the frame number $i$

$$
R_{k}=e^{-j \frac{2 \pi}{N} k\left(\bar{\theta}_{m, I}+\theta_{m, F}\right)}+W_{k}, k \in \Psi,
$$

where $W_{k}=V_{k} /\left(H_{m, k} P_{m, k}\right)$ is the complex-valued AWGN with zero mean and the variance of $\sigma^{2}$. To distinguish the correlation peaks obtained in (8), the differential integral delay $\bar{\theta}_{m, I}$ can be roughly estimated as

$\widehat{\bar{\theta}}_{m, I}=\frac{N}{2 \pi} \arg \left\{\sum_{k=0}^{Q-2} R_{k}^{*} R_{k+1}+\sum_{k=\Delta}^{\Delta+Q-2} R_{k}^{*} R_{k+1}\right\}, 0 \leq m \leq M-1$, 
where $\arg \{x\}$ denotes the angle of the complex-valued $x$.

The $M$ differential integral delays can be obtained after the processing in (13) over $M$ adjacent TDS-OFDM signal frames, whereby the TPS from only one transmitter is received in each frame. Thus the final integral TOA estimation is obtained by associating the results in (8) with the nearest counterparts in (13) as below

$$
\widehat{\theta}_{m, I}=d_{0}+\left\langle\widehat{\bar{\theta}}_{m, I},\left\{d_{m}\right\}_{m=0}^{M-1}\right\rangle, 0 \leq m \leq M-1,
$$

where $\left\langle\widehat{\bar{\theta}}_{m, I},\left\{d_{m}\right\}_{m=0}^{M-1}\right\rangle$ denotes the element of $\left\{d_{m}\right\}_{m=0}^{M-1}$ that is the nearest to $\overline{\bar{\theta}}_{m, I}$. Notice that the integral delay estimation accuracy within the range $\left(-T_{s} / 8,+T_{s} / 8\right]$ is guaranteed by the near-ideal autocorrelation property of the time-domain PN sequence, not by the results from (13).

Fractional TOA estimation using frequency-domain TPS: After compensating for the integral delay $\theta_{m, I}$, the received TPS associated with the $m$ th transmitter can be expressed as

$$
R_{k}=e^{-j \frac{2 \pi}{N} k \theta_{m, F}}+W_{k}, k \in \Psi .
$$

The fractional delay $\theta_{m, F}$ can be estimated according to

$$
\widehat{\theta}_{m, F}=\frac{N}{2 \pi \Delta} \arg \left\{\sum_{k=0}^{N-1-\Delta} R_{k}^{*} R_{k+\Delta}\right\} .
$$

The estimation range of $\widehat{\theta}_{m, F}$ in (16) is $\left(-\frac{N}{2 \Delta},+\frac{N}{2 \Delta}\right]$, which is larger than the range of the actual fractional delay $\theta_{m, F} \in$ $(-1 / 8,+1 / 8]$. The TDOA-based ranging difference between the $m$ th and $n$th transmitters is

$$
\widehat{D}_{m, n}=\widehat{D}_{m}-\widehat{D}_{n}=\left(\widehat{\theta}_{m, I}+\widehat{\theta}_{m, F}-\widehat{\theta}_{n, I}-\widehat{\theta}_{n, F}\right) T_{s} c,
$$

where $c=3 \times 10^{8} \mathrm{~m} / \mathrm{s}$ is the speed of light in the free space, $\widehat{D}_{m}$ and $\widehat{D}_{n}$ are the TOA-based ranging results for the $m$ th and $n$th transmitters, respectively.

Due to the TDM of TPS, the receiver knows that the TPS in the current received signal frame is transmitted by which transmitter, see (11). Therefore, the ranging differences obtained in (17) can be easily associated with the corresponding transmitters whose fixed locations can be pre-stored at the receiver.

Finally, the transmitter locations and the ranging differences are used for determining the receiver location using the localisation algorithms summarized in [11]. The closedform localisation algorithm proposed by Chan and Ho [12] is recommended due to its good performance in multi-path channels.

\section{TOA Based Ranging Accuracy}

Since the integral TOA can be estimated with extremely high accuracy due to the good autocorrelation property of the PN sequence, the positioning accuracy of the proposed time-frequency positioning method mainly depends on the accuracy of the fractional TOA estimation, whose variance can be derived as

$$
\operatorname{var}\left\{\widehat{\theta}_{m, F}\right\}=\frac{N}{2 \pi \Delta^{2}(N-\Delta)}\left(\frac{1}{\gamma}+\frac{1}{2 \gamma^{2}}\right),
$$

where $\gamma=\frac{1}{\sigma^{2}}$ is the average signal-to-noise ratio (SNR).

Using the results of (17) and (18), the TOA-based ranging accuracy of the proposed scheme, in terms of root mean square error (RMSE), is therefore given by

$$
\operatorname{RMSE}\left(\widehat{D}_{m}\right)=\frac{N T_{s} c}{2 \pi \Delta} \sqrt{\frac{1}{N-\Delta}\left(\frac{1}{\gamma}+\frac{1}{2 \gamma^{2}}\right)} .
$$

It should be pointed out that the positioning accuracy will depend on the above ranging accuracy as well as the localisation algorithm employed.

\section{Computational Complexity}

The correlation-based positioning method [5] has a low complexity of $O\left(N_{p}\right)$, where $N_{p}$ is the total number of used data symbols for positioning. The positioning method [6] based on the super resolution algorithm has a high complexity of $O\left(N_{p}^{3}\right)$.

For the proposed positioning scheme, the integral TOA estimation in (8) and (13) requires $N_{p}$ and $2 Q$ multiplications, respectively, while the fractional TOA estimation in (16) demands $Q+1$ multiplications. Thus the proposed positioning scheme has a low complexity of $O\left(N_{p}+Q\right)$, and it can easily be implemented in TDS-OFDM receivers.

\section{E. System Compatibility}

At the transmitter part, the TDM of TPS requires that only one transmitter sends TPS in a specific signal frame, while TPS is transmitted by every transmitter in each signal frame in conventional SFN. This trivial modification can be easily implemented by an on-off switch, without changing the current infrastructure of TDS-OFDM systems. At the receiver part, TDM of TPS has no impact on TPS detection and normal TV program reception at the receiver, since the TDM of TPS still guarantees the reception of TPS in every signal frame. Therefore, maximum backward system compatibility is achieved.

\section{Simulation Results}

Computer simulations were carried out to evaluate the performance of the proposed positioning scheme. The main system parameters were configured to be consistent with the TDS-OFDM system specified in [4]. The received signal was over-sampled by the factor of four. Four transmitters were assumed to be visible in the simulated SFN, i.e. $M=4$, and Chan and Ho's localisation algorithm [12] was used. The AWGN channel, the Indoor A multi-path channel [13] and the severe frequency-selective 8th State Administration of Radio Film and Television fading channel (SARFT 8) for Chinese DTV test [9] were used in the simulation.

Fig. 4 presents the TOA-based ranging accuracy comparison for three schemes, in terms of RMSE over the AWGN channel, where the theoretical bound (19) is also included. When the SNR was $20 \mathrm{~dB}$, the ranging error was about $2.4 \mathrm{~m}$ for Mensing's method based on correlation [5], $0.49 \mathrm{~m}$ for the MUSIC-based algorithm [6], and $0.15 \mathrm{~m}$ for the proposed scheme. It can be seen that the proposed scheme achieved 


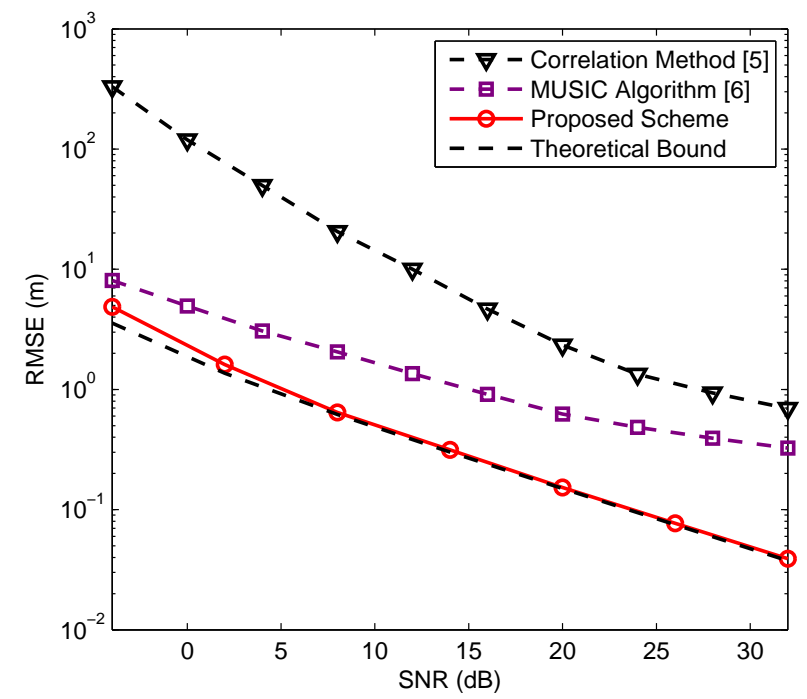

Fig. 4. Ranging accuracy comparison of three schemes over AWGN channel.

the best performance. Moreover, its ranging error approached the theoretical bound when the SNR was above $8 \mathrm{~dB}$.

Fig. 5 shows the proposed TDOA-based positioning accuracy achieved over the two multi-path channels. For reference, both the simulated positioning accuracy and the theoretical bound over the AWGN channel are included in Fig. 5, where it is seen that the ranging error of $0.15 \mathrm{~m}$ generated the positioning error of $0.082 \mathrm{~m}$ over the AWGN channel with the SNR value of $20 \mathrm{~dB}$. Under the same SNR value, the positioning accuracy was $0.092 \mathrm{~m}$ over SARFT 8 channel, and $0.067 \mathrm{~m}$ over Indoor A channel. It is interesting that a better positioning performance was obtained for Indoor A multi-path channel than for the AWGN channel. The reason for this better positioning accuracy may be explained as follows. The TPS are located at the two sides of the used subcarriers (see Fig. 2), and the CFR over these subcarriers corresponds to the low-frequency response of the channel. Indoor A channel has a good low-frequency response. This

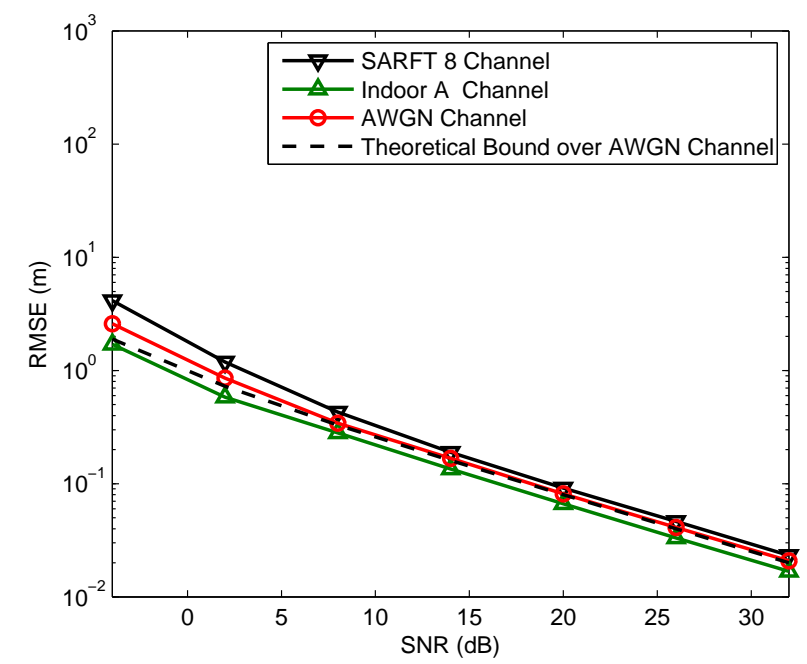

Fig. 5. Positioning accuracy of the proposed scheme over multi-path as well as AWGN channels. results in the higher equivalent SNR over the subcarrier set $k \in \Psi$ than the averaged channel SNR, thus yielding a better positioning performance.

We also compared the bit error rate performance of the conventional TDS-OFDM system with that of the TDS-OFDM system which implemented the proposed positioning scheme. No performance degradation was observed for the latter.

\section{CONCLUSION}

The positioning solution using TDS-OFDM signals for Chinese DTV standard in SFN has been proposed in this paper. The TPS embedded in the TDS-OFDM signal is time-division multiplexed as orthogonal frequency-domain pilots. The proposed time-frequency positioning scheme jointly utilises the time-domain PN sequence and frequency-domain TPS (pilots) for accurate TOA estimation of each transmitter. Results obtained have shown that the proposed positioning scheme achieves a higher positioning accuracy compared with thestate-of-art methods, while imposing a low complexity. Furthermore, it has no impact on normal TV program reception, and backward system compatibility can be maintained. The idea of time-frequency joint positioning can be also applied to other OFDM-based wireless systems.

\section{REFERENCES}

[1] M. Rabinowitz and J. Spilker, "A new positioning system using television synchronization signals," IEEE Trans. Broadcast., vol. 51, no. 1, pp. 51-61, Mar. 2005.

[2] X. Wang, Y. Wu, and J. Chouinard, "A new position location system using DTV transmitter identification watermark signals," EURASIP Journal on Applied Signal Processing, vol. 2006, pp. 1-11, 2006.

[3] D. Serant, P. Thevenon, M. L. Boucheret, O. Julien, C. Macabiau, S. Corazza, M. Dervin, and L. Ries, "Development and validation of an OFDM/DVB-T sensor for positioning," in Proc. IEEE/ION Position Location and Navigation Symposium (PLANS'10) (Indian Wells, USA), May 4-6, 2010, pp. 988-1001.

[4] Framing Structure, Channel Coding and Modulation for Digital Television Terrestrial Broadcasting System. Chinese National Standard, GB 20600-2006, Aug. 2006.

[5] C. Mensing, S. Plass, and A. Dammann, "Synchronization algorithms for positioning with OFDM communications signals," in Proc. 4th Workshop on Positioning, Navigation and Communication (Hannover), March 22, 2007, pp. 205-210.

[6] X. Li and K. Pahlavan, "Super-resolution TOA estimation with diversity for indoor geolocation," IEEE Trans. Wireless Commun., vol. 3, no. 1, pp. 224-234, Jan. 2004.

[7] A. Mattsson, "Single frequency networks in DTV," IEEE Trans. Broadcast., vol. 51, no. 4, pp. 413-422, Dec. 2005.

[8] C. yen Ong, J. Song, C. Pan, and Y. Li, "Technology and standards of digital television terrestrial multimedia broadcasting," IEEE Commun. Mag., vol. 48, no. 5, pp. 119-127, May 2010.

[9] J. Wang, Z. Yang, C. Pan, M. Han, and L. Yang, "A combined code acquisition and symbol timing recovery method for TDS-OFDM," IEEE Trans. Broadcast., vol. 49, no. 3, pp. 304-308, Sep. 2003.

[10] L. Dai, Z. Wang, J. Wang, and Z. Yang , "Positioning with OFDM signals for the next-generation GNSS," IEEE Trans. Consumer Electron. vol. 56, no. 2, pp. 374-379, May 2010.

[11] A. Sayed, A. Tarighat and N. Khajehnouri, "Network-based wireless location: challenges faced in developing techniques for accurate wireless location information," IEEE Signal Processing Magazine, vol. 22, no. 4, pp. 24-40, July 2005.

[12] Y. T. Chan and K. C. Ho, "A simple and efficient estimator for hyperbolic location," IEEE Trans. Signal Processing, vol. 42, no. 8, pp. 1905-1915, Aug. 1994.

[13] Guideline for Evaluation of Radio Transmission Technology for IMT2000. Recommendation ITU-R M.1225, 1997. 